\title{
Comparison of Anti-müllerian Hormone (AMH) and Hormonal Assays for Phenotypic Classification of Polycystic Ovary Syndrome
}

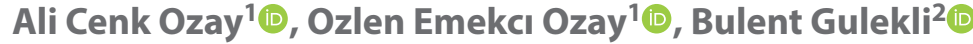 \\ ${ }^{1}$ Department of Obstetrics and Gynecology, Faculty of Medicine, Near East University, Nicosia, Cyprus \\ ${ }^{2}$ Department of Obstetrics and Gynecology, Faculty of Medicine, Dokuz Eylul University, Turkey
}

\begin{abstract}
Objectives: The aim is to compare the hormonal status and anti-müllerian hormone (AMH) levels of patients who have different polycystic ovary syndrome (PCOS) phenotypes, polycystic ovarian morphology (PCOM) and healthy women.

Material and methods: A total of 350 PCOS women, 71 women with PCOM and 79 healthy women with normal ovarian morphology (NOM) were observed. PCOS patients were divided into groups according to the phenotypes. Phenotype A- characterized by anovulation, hyperandrogenism and PCOM; phenotype B- defined as anovulation, hyperandrogenism; Phenotype C- identified as hyperandrogenism and PCOM; Phenotype D- outlined as anovulation and PCOM. AMH levels were compared for each group.

Results: Among 350 PCOS patients the highest number belonged to phenotype $A(n=117,33.4 \%)$. The rest were distrubuted as follows: phenotype $B(n=89,25.4 \%)$, phenotype $C(n=72,20.6 \%)$, phenotype $D(n=72,20.6 \%)$. Phenotype $A$ $(9.17 \pm 4.56)$ had the highest mean AMH levels in our study. Comparison of AMH levels showed a statistically significant difference between phenotypes $A$ and $D$. There was a statistically significant difference on comparison of AMH between NOM, PCOM and all PCOS phenotypes.

Conclusions: Phenotype A is the most serious form of PCOS and these patients has all three features which are hyperandrogenism, anovulation and ultrasound findings of polycystic ovary (PCO). AMH reflects the severity of PCOS and patients with Phenotype A have higher AMH levels.
\end{abstract}

Key words: polycystic ovary syndrome; phenotype; polycystic ovarian morphology; anti müllerian hormone; hyperandrogenism

Ginekologia Polska 2020; 91, 11:661-667

\section{INTRODUCTION}

Polycystic ovary syndrome (PCOS) is the highest prevalent hormonal disturbance among reproductive age women. PCOS affects $4-21 \%$ of women worldwide [1]. The symptoms of PCOS include menstrual irregularity, clinical or biochemical hyperandrogenism findings and obesity. The definition and diagnosis of PCOS have long been debated. In 1990, National Institute of Child Health and Human Disease (NICHD) congress participants agreed that the major diagnostic criteria for PCOS should include hyperandrogenism and menstrual dysfunction and the exclusion of other endocrine disorders [2]. On the other hand, the ultrasound has been widely used in Europe for the diagnosis of PCOS [3]. In 2003, a meeting was held in Rotterdam to provide consensus for PCOS diagnostic criteria. According to the Rotterdam classification in 2003, when two or more of the fol- lowing aspects are existent, PCOS can be determined: anovulation, hypernadrogenism and polycystic ovaries [4, 5]. In the Rotterdam consensus conference, it was decided that the following sonographic descriptions of polycystic ovarian morphology (PCOM) should be included: enlarged volume of the ovaries $\left(\geq 10 \mathrm{~cm}^{3}\right)$ or either $\geq 12$ follicles per ovary sized between 2-9 mm [5]. The Rotterdam consensus decisions generated the possibility of four phenotypes of PCOS. For the diagnosis of PCOS, only the presence of PCOM on ultrasound is not acceptable [5]. In addition, according to Azziz, the definition of PCOM should be made very carefully and in the abscence of any other sign or symptom of PCOS, PCOM should not be accepted as PCOS [6]. The clinical significance of the polycystic presentation of the ovaries on ultrasound still continues to be unclear. Indeed, signs and symptoms are heterogeneously combined in each affected 
woman. There is a wide spectrum of clinical and biochemical differences between PCOS patients [7]. The classification of such a varied pathology has presented dilemma for the gynecologist and therefore set up the potentiality of four different phenotypes of PCOS.

According to the Rotterdam criteria, there are four major phenotypes defined on the symptoms and clinical findings of PCOS. In patients with PCOS, phenotypes should be determined by following the correct algorithm in order to obtain healthier results [8]. Phenotype A- identified by anovulation, hyperandrogenism and polycystic ovaries on ultrasound; phenotype B- identified with anovulation, hyperandrogenism; Phenotype C- defined as hyperandrogenism and polycystic ovaries on ultrasound; Phenotype D- diagnosed as anovulation, polycystic ovaries on ultrasound [5]. The utmost serious form of PCOS is phenotype $A$ and these patients have all three features which are hyperandrogenism, anovulation and ultrasound findings of polycystic ovary (PCO).

Anti-müllerian hormone $(\mathrm{AMH})$, a polypeptide, which is synthesized from the granulosa cells of the preantral and early developing antral follicles, belongs to the transforming growth factor beta superfamily [9]. The major physiological act of AMH in the ovary is the prevention of primordial follicles recruitment and the regulation of FSH secretion in the early follicular phase [10]. Antral follicule count is associated with serum AMH levels. PCOS patients display an elevated number of antral follicles; therefore AMH levels are 2-3-fold augmented in PCOS patients [11-14]. As a result of a recent study in animals, AMH acted a significant part in the pathogenesis of PCOS starting from intrauterine life [15]. Pigny et al. [16] found that there was a significant relation between follicle count and $\mathrm{AMH}$ values in PCOS patients. Although the AMH is elevated in patients with PCOS, it is still not recommended to use serum $\mathrm{AMH}$ measurement alone to diagnose PCOS $[17,18]$. Since the phenotypes of polycystic ovary syndrome has different clinical and biochemical features, it has been claimed that the serum levels of $\mathrm{AMH}$ may vary between these phenotypes. In a recent study it is shown that serum AMH levels were higher in hyperandrogenic PCOS phenotypes and in PCOS patients that have all three Rotterdam criterias [19].

PCOS is a heterogenous clinical condition and the four major phenotypes show different clinical presentations. Investigation of the disparities between women with different phenotypes of PCOS, women with polycystic appearance only (PCOM) and healthy women with normal ovarian morphology (NOM) enhance the understanding of the pathophysiology of PCOS. Our aim is to observe and compare the hormonal status and $\mathrm{AMH}$ levels of patients with different PCOS phenotypes, PCOM and healthy women with normal ovarian morphology. Our hypothesis is that PCOS pheno- types with anovulation may produce elevated levels of AMH compared to phenotypes with regular ovulatory cycles or only polycystic ovarian morphology (PCOM) on ultrasound.

\section{MATERIAL AND METHODS}

The indicated retrospective study was planned in the Obstetrics and Gynecology Clinic of Dokuz Eylul University Hospital, Faculty of Medicine, Izmir/Turkey, during the time period of January 2012 to July 2015. The research customs was authorized by Dokuz Eylul University local review board. Enlightened approval form was collected from all participitants of the study. In date range we specified, 1871 patients who underwent serum anti-mullerian hormone tests in our hospital were identified by scanning the database and patient files of the hospital. The flow chart of the study is shown in Figure 1. A total of 500 eligiable patients were recruited, including 350 PCOS women, 71 women with polycystic ovarian morphology on ultrasound (PCOM) and 79 healthy women with normal ovarian morphology (NOM) which were suitable for our study. PCOS patients were divided into groups according to the phenotypes defined in Rotterdam criteria [5].

In our hospital, anamnesis, gynecological examination and pelvic ultrasonography are standard practice for all patients who apply to the gynecology outpatient clinic. These history and examination findings were documented from hospital database and patients'files. Calculations were done for Ferriman-Gallwey score (FGS) and Body Mass Index (BMI). Hirsutism was represented when FGS was $\geq 8$. PCOS diagnosis was made according to the Rotterdam Consensus [5]. The polycystic ovarian morphology was described as reported by the Rotterdam criteria [5, 6]. All healthy women had regular monthly menstrual cycles and initial examination by transvaginal ultrasound showed normal uteruses and ovaries. Exclusion principles were as follows; age up 35 years,

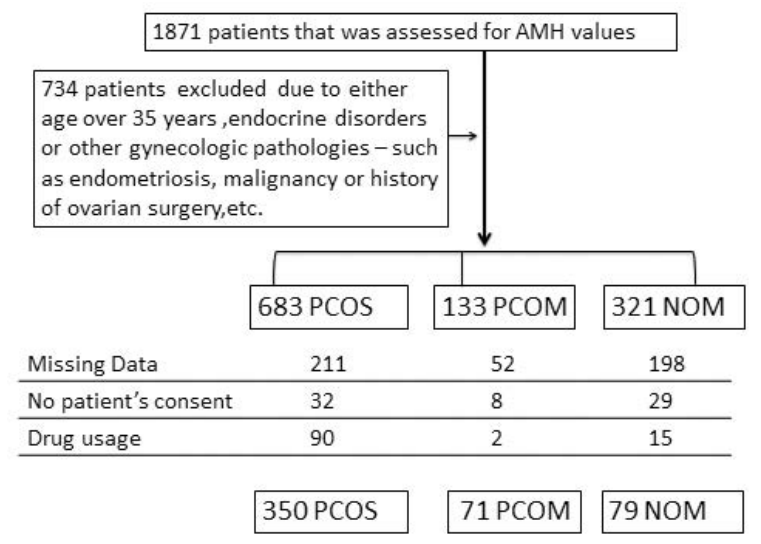

Figure 1. Flow chart of the study; PCOS - polycystic ovary syndrome; PCOM — polycystic ovarian morphology on ultrasound; NOM - normal ovarian morphology 
ovarian surgery history, thyroid disease, systemic diseases, hyperprolactinemia, congenital adrenal hyperplasia, or drug usage effecting androgen metabolism, glucose/insulin metabolism and hypothalamus-hypophysis-ovary axis. In addition, the women who had used contraceptives or hormonal treatment up to 6 months were also considered as exclusion factors. If any existence of lesions, cysts or follicles greater than $10 \mathrm{~mm}$ on the ovaries, it was accepted as an exclusion feature. Weight and height were measured in the initial check-up. The formula weight $(\mathrm{kg}) /$ height $(\mathrm{m})^{2}$ was used to analyze BMI. Biochemical and hormonal assesments were done for anti-müllerian hormone (AMH), androstenodione, thyroid stimulating hormone (TSH), luteinizing hormone (LH), total testosterone, follicle stimulating hormone (FSH), sex hormone binding globulin (SHBG), prolactin (PRL), insulin and fasting plasma glucose. Homeostatic model of insulin resistance (HOMA-IR) was used to evaluate normal insulin sensitivity. Determination of HOMA-IR was by the equation: HOMA-IR = fasting blood glucose $(\mathrm{mg} / \mathrm{dL}) \mathrm{X}$ fasting insulin $(\mathrm{mlU} / \mathrm{mL}) / 405$. Definition of insulin resistance was made in the presence of HOMA-IR being equal or greater than 2.5. Formula for the calculation of free androgen index (FAI) was 100x (Total testosterone/SHBG). Between the second and fifth days of the menstrual cycle, patients' blood samples were collected in the morning following 8 hours fasting. For amenorrhoeic women, pregnancy was eliminated then, medroxyprogesterone acetate (TARLUSAL; Deva Holding A.S. Istanbul, Turkey) was initiated $5 \mathrm{mg}$ twice a day for a 5 day period to promote uterine bleeding.

The commercial kit as reported by the manufacturer's guidance depend on the settlements of the competitive enzyme linked immunosorbent assay (ELISA) approach (catalog number: CSB-E12756h, CUSABIO Biotech Co., USA) was used to evaluate serum anti-müllerian hormone (AMH).
A basic curve of known concentration (0, 0.375, 1.31, 4.69, 28.12 , and $150 \mathrm{ng} / \mathrm{mL}$ ) of AMH was certified and the concentration of analyte in the fragments was adjusted correspondingly. The sensitivity of the ELISA assays of AMH was $0.375 \mathrm{ng} / \mathrm{mL}$; the detection range was $0.375-150 \mathrm{ng} / \mathrm{mL}$ and intraassay coefficient of variation was $<10 \%$, and interassay coefficient of variation was $<15 \%$.

Statistics are demonstrated as mean \pm standard deviation except elseways declared. Calculations were done by the program Statistical Program for Social Sciences (SPSS) version 16. (SPSS, Chicago, IL). To check for the homogeneity of the study group, the Kolmogorov Smirnov test was conducted. Continuous variables (normally distributed) were compared by one-way ANOVA (> 2 groups) and then Bonferroni test was used for post hoc comparison. Tamhane's test was performed for continous variables that were not following normal distribution. $P<0.05$ was accepted as the level of significance.

\section{RESULTS}

An overall of 500 women's results were evaluated. 350 PCOS patients, 71 women with polycystic ovarian morphology on ultrasound (PCOM) and 79 healthy women with normal ovarian morphology (NOM) were assessed. Among the 350 PCOS patients the highest number of patients belonged to phenotype $A(n=117,33.4 \%)$. The rest were distrubuted as follows: phenotype $B(n=89,25.4 \%)$, phenotype $C$ ( $n=72,20.6 \%)$, phenotype $D(n=72,20.6 \%)$. A comparison of the demographic and hormonal status of NOM, PCOM and PCOS phenotypes is shown in Table 1. The mean BMIs of all groups were similar and the mean BMI of groups were less than $25 \mathrm{~kg} / \mathrm{m}^{2}$. Comparison of the LH/FSH ratio showed that all PCOS phenotypes were found to be statistically similar. In fact, there was a statistically significant difference

\begin{tabular}{|c|c|c|c|c|c|c|c|}
\hline Variables & $\begin{array}{l}\text { NOM } \\
(n=79)\end{array}$ & $\begin{array}{l}\text { PCOM } \\
(n=71)\end{array}$ & $\begin{array}{l}P \cos A \\
(n=117)\end{array}$ & $\begin{array}{l}\text { PCOS B } \\
(n=89)\end{array}$ & $\begin{array}{l}P \cos C \\
(n=72)\end{array}$ & $\begin{array}{l}\text { PCOS D } \\
(n=72)\end{array}$ & $\mathbf{P}$ \\
\hline Age [years] & $24.05 \pm 4.59$ & $24.86 \pm 4.17$ & $23.54 \pm 4.15$ & $23.99 \pm 4.35$ & $23.40 \pm 4.84$ & $24.81 \pm 5.05$ & NS \\
\hline $\mathrm{BMI}\left[\mathrm{kg} / \mathrm{m}^{2}\right]$ & $24.70 \pm 3.35$ & $24.93 \pm 3.49$ & $24.77 \pm 4.77$ & $23.02 \pm 4.71$ & $24.03 \pm 4.48$ & $23.06 \pm 4.11$ & NS \\
\hline LH/FSH ratio & $1.01 \pm 0.42$ & $1.11 \pm 0.56$ & $1.70 \pm 1.08$ & $1.84 \pm 1.49$ & $1.44 \pm 0.72$ & $1.71 \pm 0.95$ & $<0.001^{*}, * *$ \\
\hline $\mathrm{AMH}[\mathrm{ng} / \mathrm{mL}]$ & $3.65 \pm 2.42$ & $2.99 \pm 2.00$ & $9.17 \pm 4.56$ & $8.15 \pm 4.85$ & $7.30 \pm 4.13$ & $6.18 \pm 5.46$ & $<0.001^{*, * *, a}$ \\
\hline SHBG [nmol/L] & $67.76 \pm 24.55$ & $69.52 \pm 23.29$ & $42.18 \pm 26.02$ & $46.44 \pm 24.75$ & $44.34 \pm 38.81$ & $55.73 \pm 30.81$ & $<0.001^{a, b, c}$ \\
\hline Androstenodione $[\mathrm{ng} / \mathrm{mL}]$ & $1.68 \pm 1.05$ & $1.60 \pm 0.93$ & $6.09 \pm 5.14$ & $4.56 \pm 4.04$ & $4.76 \pm 3.73$ & $1.95 \pm 0.60$ & $<0.001^{b, c, d}$ \\
\hline FAI & $1.48 \pm 0.70$ & $1.43 \pm 0.66$ & $7.28 \pm 5.37$ & $6.14 \pm 4.92$ & $6.81 \pm 5.65$ & $3.48 \pm 3.50$ & $<0.001^{*},{ }^{* *}, \mathrm{e}$ \\
\hline Total testosterone [ng/dL] & $0.90 \pm 0.32$ & $0.90 \pm 0.31$ & $2.15 \pm 0.75$ & $1.97 \pm 0.60$ & $1.87 \pm 0.66$ & $1.29 \pm 0.40$ & $<0.001^{*}$,**, e \\
\hline HOMA-IR & $1.60 \pm 1.67$ & $1.20 \pm 1.29$ & $2.91 \pm 2.31$ & $2.63 \pm 2.14$ & $2.70 \pm 2.35$ & $2.75 \pm 2.20$ & $<0.001^{*}$,** $^{* *}$ \\
\hline
\end{tabular}

*Significant difference in NOM and PCOS A, PCOS B, PCOS C, PCOS D; * Significant difference in PCOM and PCOS A, PCOS B, PCOS C, PCOS D; aSignificant difference in

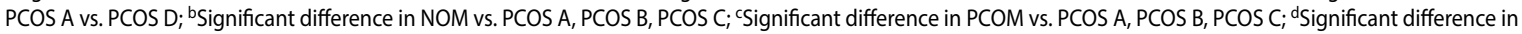
PCOS D vs. PCOS A, PCOS B, PCOS C; eSignificant difference in PCOS D vs. PCOS A, PCOS B, PCOS C, NOM, PCOM; BMI - body mass index; LH - luteinizing hormone; $\mathrm{FSH}$ - follicle stimulating hormone; Total T — total testosterone; FAI — free androgen Index; AMH — antimüllerian hormone; HOMA-IR - homeostatic model for assessment of insulin resistance; NS - nonsignificant; ${ }^{*} p<0.05$ is statistically significant. One-way ANOVA test 
between PCOM, NOM and all PCOS phenotypes in terms of the $\mathrm{LH} / \mathrm{FSH}$ ratio $(p<0.001)$. The mean highest $\mathrm{AMH}$ levels were in phenotype $A(9.17 \pm 4.56 \mathrm{ng} / \mathrm{mL})$ which was followed by phenotype $B(8.15 \pm 4.85 \mathrm{ng} / \mathrm{mL})$, phenotype $C$ $(7.30 \pm 4.13 \mathrm{ng} / \mathrm{mL})$, and phenotype $D(6.18 \pm 5.46 \mathrm{ng} / \mathrm{mL})$. Comparison of AMH levels showed a statistically significant difference between PCOS phenotypes A and D. Table 2 demonstrates the AMH levels of each group. There was a statistically significant difference on comparison of $\mathrm{AMH}$ between NOM, PCOM and all PCOS phenotypes (Fig. 2). A graphic of free androgen index is shown in Figure 3. In the comparison of HOMA-IR, there was no statistically significant difference

\begin{tabular}{|l|l|l|l|l|l|}
\multicolumn{6}{|}{ Table 2. AMH levels of groups } \\
\hline Phenotype & OA & HA & PCO & Frequency [\%] & AMH [ng/mL] \\
\hline A & + & + & + & $\% 23.4(n=117)$ & $9.17 \pm 4.56$ \\
\hline B & + & + & - & $\% 17.8(n=89)$ & $8.15 \pm 4.85$ \\
\hline C & - & + & + & $\% 14.4(n=72)$ & $7.30 \pm 4.13$ \\
\hline D & + & - & + & $\% 14.4(n=72)$ & $6.18 \pm 5.46$ \\
\hline PCOM & - & - & + & $\% 14.2(n=71)$ & $2.99 \pm 2.00$ \\
\hline NOM & - & - & - & $\% 15.8(n=79)$ & $3.65 \pm 2.42$ \\
\hline
\end{tabular}

$\mathrm{OA}$ - oligoanovulation; $\mathrm{HA}$ - hyperandrogenism; $\mathrm{PCO}$ - polycystic ovary appearance; $\mathrm{AMH}$ - anti müllerian hormone; $\mathrm{PCOM}$; polycystic ovarian morphology; NOM — normal ovarian morphology

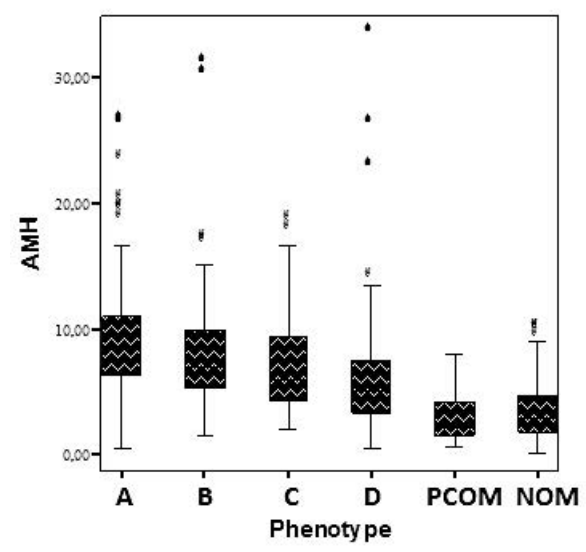

NOM vs PCOM $p=0.65$

NOM vs PCOS phenotype $D \boldsymbol{p}=\mathbf{0 . 0 0 7}$

NOM vs PCOS phenotype $A, B, C \boldsymbol{p}<\mathbf{0 . 0 0 1}$

PCOM vs PCOS all phenotypes $\boldsymbol{p}<\mathbf{0 . 0 0 1}$

PCOS A vs B $p=0.997$

PCOS A vs $C p=0.968$

$P C O S A$ vs $D p=\mathbf{0 . 0 0 2}$

PCOS B vs $C p=0.663$

PCOS B vs D $p=1.0$

$P \operatorname{COS} C$ vs $D p=0.442$

Figure 2. Mean values of AMH for PCOS phenotypes, PCOM and NOM; NOM — normal ovarian morphology; PCOM - polycystic ovarian morphology on ultrasound; PCOS - polycystic ovary syndrome between PCOS phenotypes. HOMA-IR showed a statistically significant difference between PCOM and all phenotypes of PCOS patients. NOM participants showed markedly lower HOMA-IR levels compared to all PCOS phenotypes (Fig. 4).

\section{DISCUSSION}

We investigated the clinical, hormonal and $\mathrm{AMH}$ levels and this study maintains knowledge on the diversity in these factors among the four phenotypes of PCOS according to the Rotterdam criteria [5], PCOM and healthy women with normal menstrual cycle. Mostly, AMH is produced from the small antral follicles and it's levels in circulation resemble to total antral follicle count, granulosa cell action and ovarian volume [20]. Our research supported previous studies in terms of high serum AMH levels in PCOS women compared to healthy ones and women with polycystic ovarian morphology only $[14,16,19-21]$. Ovulatory disfunctions in PCOS are generated by two mechanisms [22]. Firstly, there is an increased early follicular growth which results in an increased number of follicles. Secondly, there is an abnormal pick of dominant follicle from this increased follicular pool, leading to follicular cessation [22]. It is also known that the excess $\mathrm{AMH}$ origination by polycystic ovaries is an outcome of the increased follicle number [22]. Bhide et al. [23] suggested that for the assesment of antral follicle pool, AMH is a good tool because serum AMH concentrations have a powerful link with the antral follicle count (AFC). Although AMH is a good indicator in testing ovarian reserve, there is not enough data to determine a cut off value in PCOS diagnosis and dif-

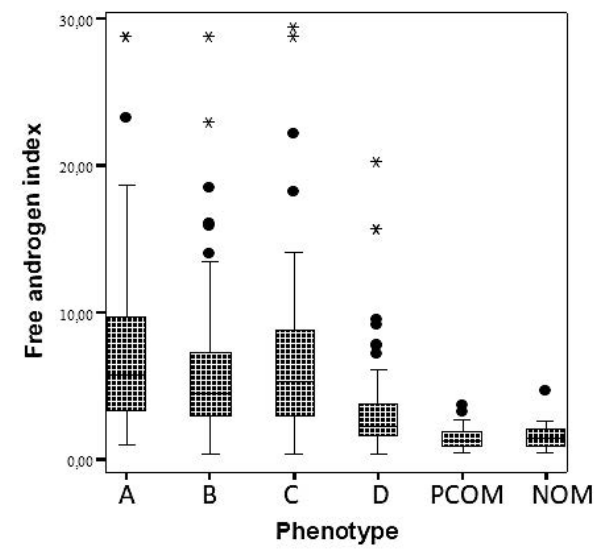

NOM vs all PCOS phenotypes $\boldsymbol{p}<\mathbf{0 . 0 0 1}$

PCOM vs all PCOS phenotypes $\boldsymbol{p}<\mathbf{0 . 0 0 1}$

PCOS D vs $A, \boldsymbol{p}<\mathbf{0 . 0 0 1}$

PCOS D vs B $\boldsymbol{p}<0.001$

PCOS D vs $C p<0.001$

Figure 3. Mean values of free androgen index for PCOS phenotypes, PCOM and NOM; NOM - normal ovarian morphology; PCOM - polycystic ovarian morphology on ultrasound; PCOS - polycystic ovary syndrome 


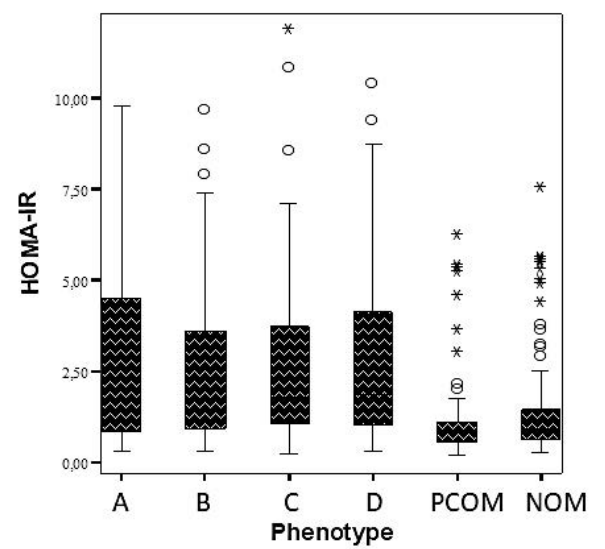

NOM vs all PCOS phenotypes $\boldsymbol{p}<\mathbf{0 . 0 0 1}$

NOM vs PCOM $p=0.793$

PCOM vs PCOS all phenotypes $\boldsymbol{p}<\mathbf{0 . 0 0 1}$

Figure 4. Mean values of HOMA-IR for PCOS phenotypes, PCOM and NOM; NOM - normal ovarian morphology; PCOM - polycystic ovarian morphology on ultrasound

ferentiating between the phenotypes of PCOS [17, 18, 24]. The relation between $\mathrm{AFC}$ and $\mathrm{AMH}$ predicts that $\mathrm{AMH}$ values may be higher in PCOM than NOM, but in our study, serum AMH values were similar for PCOM and NOM group. This situation shows that the increased number of 2-9 mm diameter follicles, is not the only determinant of serum AMH [2]. $\mathrm{LH}$ is also involved in higher AMH production [25]. When granulosa cells are exposed to $\mathrm{LH}$, there is an increased secretion of $\mathrm{AMH}$ in anovulatory polycystic ovaries, which is not seen in normal ovaries [25]. In our study group, AMH levels were statistically lower in PCOM patients compared to all phenotypes of PCOS. In our study, the most common PCOS phenotype was $A$, similar to previous studies in the literature [26-28]. In the current study, hyperandrogenic PCOS phenotypes $(A, B, C)$ presented higher AMH levels as compared with normoandrogenic phenotype $D$. The highest $\mathrm{AMH}$ levels were observed in phenotype $A$ which shows all three components of the syndrome. AMH levels of PCOS phenotype A were statistically significantly higher compared to PCOS phenotype D. Piouka et al. showed that anti-müllerian hormone levels indicate the severity of PCOS, which was negatively correlated with $\mathrm{BMI}$, and they found that phenotype $A$ has the highest levels of $A M H$ [29]. In addition, Piouka et al. [29] determined that AMH concentrations were $65 \%$ lessened in women with high BMI compared to women of normal BMI and this outcome was supported by the study of Freeman et al. [30]. Our study group was formed from patients with similar mean BMls and therefore we did not observe any correlation between BMI and $\mathrm{AMH}$.

In our study, the highest FAl was detected in Phenotype A, while the lowest value was observed in phenotype $D$ among
PCOS patients. There was a statistically significant difference in FAI between phenotype D vs other PCOS phenotypes. In addition, in the phenotype $D$ group, although the mean of FAl was in the normal range, it was significantly higher than the NOM and PCOM groups. Romualdi et al. found a markedly difference in terms of FAl, between phenotype $A$ vs phenotype $D$ and phenotype A vs healthy controls, similar to our study findings [31]. However, in contrast with our results, they did not find a statistically significant difference between phenotype D vs phenotype B and C [31]. Polak's study showed that the mean of FAl in hyperandrogenic phenotypes of PCOS was markedly higher than control group while phenotype $D$ and control groups were similar [32]. In the comparison of PCOS subgroups in terms of FAI, there was a significant increase only in phenotype A compared to the phenotype $D$ [32]. Whereas in our research, FAl was significantly higher in hyperandrogenic PCOS phenotypes $(A, B, C)$ than in phenotype $D$.

Although, insulin resistance (IR) has been very popular in the evaluation of PCOS, it is not included in the diagnostic criteria just like AMH. High insulin resistance in women with PCOS is considered as an alternate factor to assess the severity of the disease. Therefore, in most severe phenotype, insulin resistance is expected to be higher. We found that HOMA-IR values were statistically higher in all PCOS phenotypes compared to PCOM and NOM. Between the phenotypes, phenotype $A$ had the highest HOMA-IR level, but this finding was not statistically significant. We can conduct that the HOMA-IR values have same distribution among the four phenotypes. Gupta et al. observed the relationship between insulin resistance, $\mathrm{AMH}$ and $\mathrm{BMI}$ among the four phenotypes of PCOS and their study results showed no differences in terms of IR among the different phenotypes of $\mathrm{PCOS}$ [33]. About the relation between IR and $\mathrm{AMH}$, there is no concensus with some declaring a positive correlation [28, 34], while others stating there is no association [27, 29], or a negative correlation [35]. Piouka et al. found that IR indexes and AMH does not have any direct link [29]. Also, our study outcomes did not show any correlation between AMH and HOMA-IR. This result may be due to our sample's similar BMI values.

The most important strength of this study is that it is one of the largest studies in the literature that evaluates serum AMH among PCOS phenotypes. The homogenous study population and well defined PCOS, PCOM patients and healthy control groups are the advantages of this work. Although the similar characteristics of PCOS and healthy groups in terms of body mass index makes the study more homogeneous, the lack of comparison in obese PCOS patients can be considered as the limitation of the study. The other limitation of the study is that it was performed retrospectively. Additionally, the ultrasound examinations of the 
patients were done by different clinicians and this is another limitation to the study.

\section{CONCLUSIONS}

In conclusion, according to our results, the prevalence of the four phenotypes of PCOS varies and Phenotype $A$ is the most severe and common form. The literature shows controversial results on the relationship between hormonal and metabolic aspects in various phenotypes. $\mathrm{AMH}$ reflects the severity of the disease with those exhibiting all the symptoms of PCOS. Phenotype A had the highest AMH levels among the PCOS phenotypes. We could not find any correlation between AMH levels and HOMA-IR and BMI in our study.

\section{Acknowledgements}

No financial support from any organization was announced by the authors of the study. All primary data was fully controlled by the authors. The patients that were recruited for the study signed written approval form.

\section{Conflict of interest}

The authors report no potential conflict of interest.

\section{REFERENCES}

1. Lizneva D, Suturina L, Walker W, et al. Criteria, prevalence, and phenotypes of polycystic ovary syndrome. Fertil Steril. 2016; 106(1): 6-15, doi: 10.1016/j.fertnstert.2016.05.003, indexed in Pubmed: 27233760.

2. Zawadzki JK, Dunaif A. Diagnostic criteria for polycystic ovary syndrome: towards a rational approach. In: Dunaif A, Givens JR, Haseltine FP, et al. ed. Polycystic ovary syndrome. Blackwell Scientific Publications, Boston 1992: 377e84.

3. Conway GS. Polycystic ovary syndrome: clinical aspects. Baillieres Clin Endocrinol Metab. 1996; 10(2): 263-279, doi: 10.1016/s0950-351x(96)80113-3, indexed in Pubmed: 8773748.

4. Azziz R, Carmina E, Dewailly D, et al. Androgen Excess Society. Positions statement: criteria for defining polycystic ovary syndrome as a predominantly hyperandrogenic syndrome: an Androgen Excess Society guideline. J Clin Endocrinol Metab. 2006; 91(11): 4237-4245, doi: 10.1210/jc.2006-0178, indexed in Pubmed: 16940456.

5. Rotterdam ESHRE/ASRM-Sponsored PCOS Consensus Workshop Group, Rotterdam ESHRE/ASRM-Sponsored PCOS consensus workshop group. Revised 2003 consensus on diagnostic criteria and long-term health risks related to polycystic ovary syndrome (PCOS). Hum Reprod. 2004; 19(1): 41-47, doi: 10.1093/humrep/deh098, indexed in Pubmed: 14688154.

6. Azziz R. Polycystic Ovary Syndrome. Obstet Gynecol. 2018; 132(2): 321-336, doi: 10.1097/AOG.0000000000002698, indexed in Pubmed: 29995717.

7. Pasquali R, Stener-Victorin E, Yildiz BO, et al. PCOS Forum: research in polycystic ovary syndrome today and tomorrow. Clin Endocrinol (Oxf). 2011; 74(4): 424-433, doi: 10.1111/j.1365-2265.2010.03956.x, indexed in Pubmed: 21158892.

8. Azziz R, Kintziger K, Li R, et al. Recommendations for epidemiologic and phenotypic research in polycystic ovary syndrome: an androgen excess and PCOS society resource. Hum Reprod. 2019; 34(11): 2254-2265, doi: 10.1093/humrep/dez185, indexed in Pubmed: 31751476.

9. Pellatt L, Hanna L, Brincat M, et al. Granulosa cell production of anti-Müllerian hormone is increased in polycystic ovaries. J Clin Endocrinol Metab. 2007; 92(1): 240-245, doi: 10.1210/jc.2006-1582, indexed in Pubmed: 17062765.

10. La Marca A, Broekmans FJ, Volpe A, et al. ESHRE Special Interest Group for Reproductive Endocrinology--AMH Round Table. Anti-Mullerian hormone (AMH): what do we still need to know? Hum Reprod. 2009; 24(9):
2264-2275, doi: 10.1093/humrep/dep210, indexed in Pubmed: 19520713.

11. Piltonen T, Morin-Papunen L, Koivunen $\mathrm{R}$, et al. Serum anti-Müllerian hormone levels remain high until late reproductive age and decrease during metformin therapy in women with polycystic ovary syndrome. Hum Reprod. 2005; 20(7): 1820-1826, doi: 10.1093/humrep/deh850, indexed in Pubmed: 15802325.

12. Nardo LG, Yates $A P$, Roberts $S A$, et al. The relationships between $A M H$, androgens, insulin resistance and basal ovarian follicular status in non-obese subfertile women with and without polycystic ovary syndrome. Hum Reprod. 2009; 24(11): 2917-2923, doi: 10.1093/humrep/dep225, indexed in Pubmed: 19617605.

13. Pellatt L, Rice $S$, Mason HD. Anti-Müllerian hormone and polycystic ovary syndrome: a mountain too high? Reproduction. 2010; 139(5): 825-833, doi: 10.1530/REP-09-0415, indexed in Pubmed: 20207725.

14. Pigny $P$, Merlen $E$, Robert $Y$, et al. Elevated serum level of anti-mullerian hormone in patients with polycystic ovary syndrome: relationship to the ovarian follicle excess and to the follicular arrest. J Clin Endocrinol Metab. 2003; 88(12): 5957-5962, doi: 10.1210/jc.2003-030727, indexed in Pubmed: 14671196.

15. Tata B, Mimouni NE, Barbotin AL, et al. Elevated prenatal anti-Müllerian hormone reprograms the fetus and induces polycystic ovary syndrome in adulthood. Nat Med. 2018; 24(6): 834-846, doi: 10.1038/s41591-018-0035-5, indexed in Pubmed: 29760445.

16. Pigny $P$, Jonard $S$, Robert $Y$, et al. Serum anti-Mullerian hormone as a surrogate for antral follicle count for definition of the polycystic ovary syndrome. J Clin Endocrinol Metab. 2006; 91(3): 941-945, doi: 10.1210/jc.2005-2076, indexed in Pubmed: 16368745.

17. Teede HJ, Misso ML, Costello MF, et al. International PCOS Network. Recommendations from the international evidence-based guideline for the assessment and management of polycystic ovary syndrome. Fertil Steril. 2018; 110(3):364-379, doi: 10.1016/j.fertnstert.2018.05.004, indexed in Pubmed: 30033227.

18. Teede $\mathrm{H}$, Misso M, Tassone EC, et al. Anti-Müllerian Hormone in PCOS: A Review Informing International Guidelines. Trends Endocrinol Metab. 2019; 30(7): 467-478, doi: 10.1016/j.tem.2019.04.006, indexed in Pubmed: 31160167.

19. Sova $\mathrm{H}$, Unkila-Kallio $\mathrm{L}$, Tiitinen $\mathrm{A}$, et al. Hormone profiling, including anti-Müllerian hormone (AMH), for the diagnosis of polycystic ovary syndrome (PCOS) and characterization of PCOS phenotypes. Gynecol Endocrinol. 2019; 35(7):595-600, doi: 10.1080/09513590.2018.1559807, indexed in Pubmed: 30668196.

20. Iliodromiti S, Kelsey TW, Anderson RA, et al. Can anti-Mullerian hormone predict the diagnosis of polycystic ovary syndrome? A systematic review and meta-analysis of extracted data. J Clin Endocrinol Metab. 2013; 98(8): 3332-3340, doi: 10.1210/jc.2013-1393, indexed in Pubmed: 23775353.

21. Siow $Y$, Kives $S$, Hertweck $P$, et al. Serum Müllerian-inhibiting substance levels in adolescent girls with normal menstrual cycles or with polycystic ovary syndrome. Fertil Steril. 2005; 84(4): 938-944, doi: 10.1016/j.fertnstert.2005.02.052, indexed in Pubmed: 16213847.

22. Jonard S, Dewailly D. The follicular excess in polycystic ovaries, due to intra-ovarian hyperandrogenism, may be the main culprit for the follicular arrest. Hum Reprod Update. 2004; 10(2): 107-117, doi: 10.1093/humupd/dmh010, indexed in Pubmed: 15073141.

23. Bhide $P$, Homburg R. Anti-Müllerian hormone and polycystic ovary syndrome. Best Pract Res Clin Obstet Gynaecol. 2016; 37: 38-45, doi: 10.1016/j.bpobgyn.2016.03.004, indexed in Pubmed: 27103234.

24. Bozdag G, Mumusoglu S, Coskun ZY, et al. Anti-Müllerian hormone as a diagnostic tool for PCOS under different diagnostic criteria in an unselected population. Reprod Biomed Online. 2019; 39(3): 522-529, doi: 10.1016/j.rbmo.2019.04.002, indexed in Pubmed: 31182353.

25. Pierre A, Peigné $M$, Grynberg $M$, et al. Loss of $L H$-induced down-regulation of anti-Müllerian hormone receptor expression may contribute to anovulation in women with polycystic ovary syndrome. Hum Reprod. 2013; 28(3): 762-769, doi: 10.1093/humrep/des460, indexed in Pubmed: 23321213.

26. Amini P, Omani-Samani R, Hosseini R, et al. A cross-sectional comparison of clinical and endocrine parameters among phenotypes of polycystic ovarian syndrome in iranian population. Middle East Fertility Society Journal. 2018; 23(4): 425-430, doi: 10.1016/j.mefs.2018.07.005.

27. Sahmay S, Aydogan Mathyk B, Sofiyeva N, et al. Serum AMH levels and insulin resistance in women with PCOS. Eur J Obstet Gynecol Reprod Biol. 2018; 224: 159-164, doi: 10.1016/j.ejogrb.2018.03.007, indexed in Pubmed: 29605710. 
28. Wiweko $B$, Indra I, Susanto $C$, et al. The correlation between serum AMH and HOMA-IR among PCOS phenotypes. BMC Res Notes. 2018; 11(1): 114, doi: 10.1186/s13104-018-3207-y, indexed in Pubmed: 29426356.

29. Piouka A, Farmakiotis D, Katsikis I, et al. Anti-Mullerian hormone levels reflect severity of PCOS but are negatively influenced by obesity: relationship with increased luteinizing hormone levels. Am J Physiol Endocrinol Metab. 2009; 296(2): E238-E243, doi: 10.1152/ajpendo.90684.2008, indexed in Pubmed: 18957615

30. Freeman EW, Gracia CR, Sammel MD, et al. Association of anti-mullerian hormone levels with obesity in late reproductive-age women. Fertil Steril. 2007; 87(1): 101-106, doi: 10.1016/j.fertnstert.2006.05.074, indexed in Pubmed: 17109858.

31. Romualdi D, Di Florio C, Tagliaferri V, et al. The Role of Anti-Müllerian Hormone in the Characterization of the Different Polycystic Ovary Syndrome Phenotypes. Reprod Sci. 2016; 23(5): 655-661, doi: 10.1177/1933719115611751, indexed in Pubmed: 26718304

32. Polak AM, Adamska A, Krentowska A, et al. Body Composition, Serum Concentrations of Androgens and Insulin Resistance in Different
Polycystic Ovary Syndrome Phenotypes. J Clin Med. 2020; 9(3), doi: 10.3390/jcm9030732, indexed in Pubmed: 32182752.

33. Gupta M, Yadav R, Mahey R, et al. Correlation of body mass index $(\mathrm{BMI})$, anti-mullerian hormone (AMH), and insulin resistance among different polycystic ovary syndrome (PCOS) phenotypes - a cross-sectional study. Gynecol Endocrinol. 2019; 35(11): 970973, doi: 10.1080/09513590.2019.1613640, indexed in Pubmed: 31081410.

34. Skałba $P$, Cygal $A$, Madej $P$, et al. Is the plasma anti-Müllerian hormone (AMH) level associated with body weight and metabolic, and hormonal disturbances in women with and without polycystic ovary syndrome? Eur J Obstet Gynecol Reprod Biol. 2011; 158(2): 254-259, doi: 10.1016/j. ejogrb.2011.06.006, indexed in Pubmed: 21752527.

35. Chen MJ, Yang WS, Chen CL, et al. The relationship between anti-Mullerian hormone, androgen and insulin resistance on the number of antral follicles in women with polycystic ovary syndrome. Hum Reprod. 2008; 23(4): 952-957, doi: 10.1093/humrep/den015, indexed in Pubmed: 18256110 